\title{
Screening Surgeons for HIV Infection: Assessment of a Potential Public Health Program
}

\section{Citation}

Schulman, Kevin A., Robert C. McDonald, Lorna A. Lynn, lan Frank, Nicholas A. Christakis, and Sanford Schwartz. 1994. Screening surgeons for HIV infection: Assessment of a potential public health program. Infection Control and Hospital Epidemiology 15, no. 3: 147-155.

\section{Published Version}

http://dx.doi.org/10.1086/646882

\section{Permanent link}

http://nrs.harvard.edu/urn-3:HUL.InstRepos:3320926

\section{Terms of Use}

This article was downloaded from Harvard University's DASH repository, and is made available under the terms and conditions applicable to Other Posted Material, as set forth at http:// nrs.harvard.edu/urn-3:HUL.InstRepos:dash.current.terms-of-use\#LAA

\section{Share Your Story}

The Harvard community has made this article openly available.

Please share how this access benefits you. Submit a story.

\section{Accessibility}




\section{SCREENING SURGEONS FOR HIV INFECTION: Assessment of a Potential Public Health Program}

Kevin A. Schulman, MD; Robert C. McDonald, MD; Lorna A. Lynn, MD; Ian Frank, MD; Nicholas A Christakis, MD, MPH; J. Sanford Schwartz, MD

ABSTRACT

OBIECTIVE: To develop a model to assess the impact of a program of testing surgeons for human immunodeficiency virus (HIV) on the risk of HIV acquisition by their patients.

DESIGN: A Monte Carlo simulation model of physician-to-patient transmission of human immunodeficiency virus (HIV) infection using three different rates of physician-to-patient transmission per percutaneous exposure event $(0.15 \%$, $0.3 \%, 0.6 \%$ ). Data from the model were developed from a review of the medical literature and from subjective probability estimates when data were not available. We used this model to estimate on a national basis the annual number of cases of HIV transmission from surgeons to patients with and without surgeon testing and practice limitations.

RESULTS: The annual number of transmitted cases would range from $0.5( \pm 0.3)$, assuming a surgeon HIV prevalence of $0.1 \%$ and a surgeon-topatient transmission rate of $0.15 \%$, to 36.9

\section{INTRODUCTION}

The apparent transmission of human immunodeficiency virus (HIV) from an infected dentist to five of
( \pm 11.6$)$, assuming a surgeon HIV prevalence of $\mathbf{2} \%$ and a surgeon-to-patient transmission rate of $0.6 \%$. After one screening cycle, a mandatory screening program would be expected to reduce the annual transmissions to $0.05( \pm 0.03)$ and $3.1( \pm 1.1)$, respectively.

CONCLUSION: Patients are at low risk of acquiring HIV infection from an infected physician during an invasive procedure. The pqtential costs of such a program extended beyond the costs of testing and counseling. In communities with high HIV prevalence, screening surgeons and limiting their practices may decrease patient access to care. $\boldsymbol{A}$ disability insurance program also would be required to protect surgeons and trainees performing invasive procedures. Screening surgeons for HIV infection would be a costly undertaking that would reduce but not completely eliminate this risk (Infect Control Hosp Epidemiol 1994;15:147-155).

From the Clinical Economics Research Unit, Division of General Intemal Medicine, Georgetown University Medical Center (Dr. Schuiman), Washington, DC, and the Division of General Intemal Medicine (Drs. Lynn, Christakis, and Schwartz) and Section of Infectious Diseases (Dr. Frank), Department of Medicine (Drs. Lynn, Frank, Christakis, and Schwartz), the Wharton School (Drs. McDonald and Schwartz), the Robert Wood Johnson Clinical Scholars Program (Dr. Christakis), and the Leonard Davis Institute of Health Economics (Drs. Christakis and Schwartz), the University of Pennsylvania, Philadelphia, Fennsylvania.

The authors thank Henry Glick, MA, José Escarce, MD, Anand Desai, PhD, and Jason Lee for their help in developing the physician screening model, and Brad Rosenstein for his help in preparing the manuscript.

Address reprint requests to Kevin A. Schulman, MD, Clinical Economics Research Unit, Georgetown University Medical Center, 4000 Reservoir Rd. NW, Building D, Room 392, Washington, DC 20007.

93-OA-107. Schulman KA, McDonald RC, Lynn LA, Frank I, Christakis NA, Schwartz JS. Screening surgeons for HIV infection: assessment of a potential public health program. Infect Control Hosp Epidemiol 1994;15:147-155. 
TABLE 1

Model Inruts for Monte Carlo Simulation

\begin{tabular}{|c|c|c|c|}
\hline Model Parameter & Model Value & Distribution & Source \\
\hline \multicolumn{4}{|l|}{ Single Data Points: } \\
\hline Estimated inpatient HIV prevalence & $0.9 \%$ & & $(22)$ \\
\hline \multicolumn{4}{|l|}{ Annual percutaneous occupational blood exposures } \\
\hline Attendings & 3.1 & & $(26,2)$ \\
\hline Residents & 4.2 & & $(25)$ \\
\hline Medical students & 1.3 & & (25) \\
\hline \multicolumn{4}{|l|}{ Surgical professionals } \\
\hline Attendings & 100,205 & & (29) \\
\hline Residents & 21,818 & & (29) \\
\hline Graduating medical students & 2,434 & & $(30)$ \\
\hline Risk modification factor & 0.59 & & $(31)$ \\
\hline \multicolumn{4}{|l|}{ Continuous Data Points: Objective } \\
\hline $\begin{array}{l}\text { Risk of surgeon seroconversion following } \\
\text { percutaneous exposure to infected blood }\end{array}$ & $0.3 \%\left(\mathrm{Cl}_{95}=0.1 \%\right.$ to $\left.0.7 \%\right)$ & Poisson & (28) \\
\hline Duration of window period & 2.1 months $\left(\mathrm{Cl}_{95}=0\right.$ to 5.8$)$ & Log-Normal & $(37)$ \\
\hline \multicolumn{4}{|l|}{ Continuous Data Points: Subjective } \\
\hline First ELISA sensitivity & $98.5 \%$ (range $97 \%$ to $100 \%$ ) & Normal & (35) \\
\hline First ELISA specificity & $99.5 \%$ (range $99.4 \%$ to $99.6 \%$ ) & Normal & $(35)$ \\
\hline Second ELISA sensitivity & $98.5 \%$ (range $97 \%$ to $100 \%$ ) & Normal & (35) \\
\hline Second ELISA specificity & $60 \%$ (range $50 \%$ to $70 \%$ ) & Normal & $(35)$ \\
\hline Western blot sensitivity & $96 \%$ (range $94.5 \%$ to $97.5 \%$ ) & Normal & (35) \\
\hline Western blot specificity & $99.4 \%$ (range $99.3 \%$ to $99.5 \%$ ) & Normal & (35) \\
\hline Incidence conversion ratio (author's estimate) & 8 (range 4 to 12 ) & Normal & \\
\hline \multicolumn{4}{|l|}{ Multiṕle Discrete Data Points: } \\
\hline Prevalence (author's estimate) & $\begin{array}{c}0.1 \%, 0.25 \%, 0.5 \%, 0.75 \% \\
1 \%, 2 \%\end{array}$ & & \\
\hline Surgeon-to-patient transmission rate (author's estimate) & $0.15 \%, 0.3 \%, 0.6 \%$ & . & \\
\hline
\end{tabular}

clinical activities of HIV-infected healthcare providers, especially those who perform invasive procedures. Most professional organizations, including the American College of Physicians and the American Medical Association, ${ }^{4-6}$ support voluntary practice limitations, whereas several federal and state legislators have proposed mandatory HIV testing of surgeons who perform invasive procedures, with mandatory practice limitations for those who test positive. ${ }^{7-13}$ Legislation proposed in the U.S. Congress includes a provision that certain practicing HIV-infected physicians would be subject to large fines and imprisonment if they did not notify patients of their HIV status. ${ }^{13}$

The debate over screening surgeons for HIV infection has proceeded with incomplete data and, until recently, without quantitative analysis. ${ }^{14-18}$ At this time, there are three major areas of controversy: 1) the risk of patients' acquiring HIV from infected surgeons; 2) the reduction in physician-to-patient HIV transmission as a result of a program of screening surgeons for HIV infection; and 3) the costs and consequences of implementing a mandatory HIV screening program for surgeons on practice patterns and patient access to care. Data that might resolve these questions are not fully available.

This article describes our analysis of the public health ramifications of a program that annually screens all surgeons in the United States for HIV infection. We used decision analytic techniques to model HIV transmission between surgeons and their patients in order to estimate the risk to. surgeons and their patients of acquiring HIV infection in the healthcare setting. Decision analysis is a useful means of interpreting the limited data available for this public health proposal. It offers a comprehensive approach to the analysis of the clinical problem, and yet it is flexible enough to allow us to assess how the uncertainty surrounding current data estimates affect the results of the analysis.

Finally, we will discuss the public health and the broader public policy implications of instituting mandatory screening of surgeons for HIV infection 
based on the analysis we present in this article.

\section{METHODS \\ Overview}

We developed a Monte Carlo simulation model of physician-to-patient transmission of HIV infection to assess the risk of HIV transmission from surgeons to patients and the potential for a screening program to decrease this risk. Data for this model were derived from a review of the literature and from subjective probability estimates when data were not available. Multiple runs of the model were performed to assess the impact of alternate subjective assumptions on the rate of transmission of HIV infection from surgeons to patients and to test the stability of the transmission model results to changes in the assumptions used in the analysis. In this model and in our subsequent discussion, we assumed that surgeons identified as infected with the HIV virus subsequently would be excluded from performing invasive procedures (practice limitations).

\section{Transmission and Screening Model}

We used Monte Carlo simulation to develop a model for HIV transmission and screening. Monte Carlo simulation is a method of decision modeling that is used to model complex situations characterized by incomplete data. The simulation approach was used to address this problem to help understand any potential interaction among key data points and to develop variance estimates for the results of our analysis. Monte Carlo simulation requires the computer to draw inputs for each run of the model randomly from the defined distribution for each input variable. The model is based on the data elements presented in Table 1: the factors that relate to the risk of HIV transmission from surgeons to patients and to the effectiveness of a surgeon screening program to reduce this risk.

In our model, each screening cycle is conducted simultaneously for all surgeons in the United States. A result based upon four years of screening of all surgeons represents one run of the model. Data elements for each run of the model are described in Table 1. Single data points were taken as fixed values in each run of the model. At the start of each run, values were selected randomly for each of the continuous data points based on the properties of the distributions of these parameters. Results were calculated for each of the multiple discrete data points for each run of the model.

The number of incident cases among surgeons for a given year is the sum of the number of incident HrV-positive residents and attending surgeons plus the number of prevalent and incident cases among

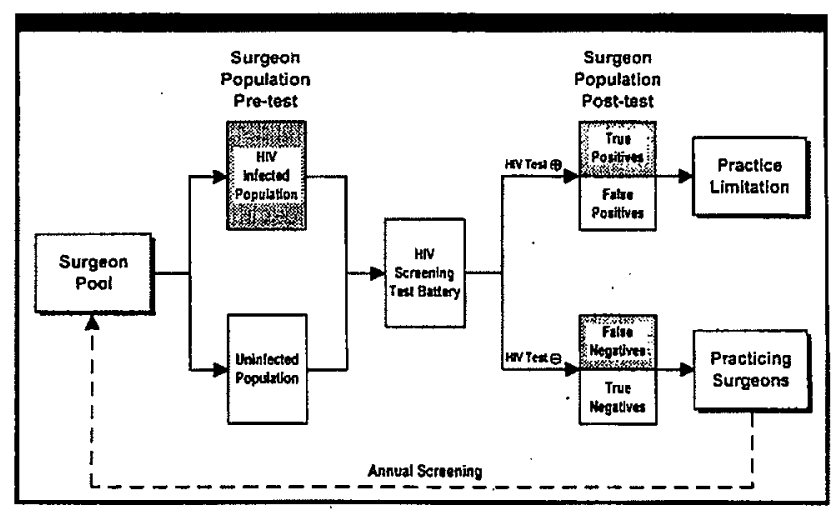

FICURE. Surgeon model used as the basis for this analysis. Surgeons in shaded areas potentially are able to transmit the HIV virus to patients.

new residents (graduating medical students entering postgraduate surgical training). Surgeons with a truenegative or false-negative test were retested in the subsequent year. Surgeons with a true-positive or false-positive test were removed from the population and not included in subsequent cycles (Figure).

The model follows the population of surgeons in the U.S. through four annual screening cycles. A four-year time frame is used because at that point the model reaches equilibrium (the estimated number of transmissions to patients remains stable despite additional testing). The transmission of HIV infection from surgeons to patients for an unscreened surgeon population is calculated for the first year of the analysis.

\section{Simulation Parameters}

The Monte Carlo simulation is based on probability distributions of model parameters when the distributions are known. For variables with unknown distributions, we developed subjective probability distributions using the range of parameter estimates reported in the literature as the limits of the distribution. When a normal distribution was assumed, the reported range was truncated to allow uniform distribution around a best-case estimate that served as the mean. The standard deviation $(\sigma)$ was calculated from the following formula ${ }^{19}$ :

$$
\sigma=\frac{.5 \cdot(\text { range })}{3}
$$

The Poisson distribution was used to approximate the binomial distribution for the risk of transmission following percutaneous exposure. We modeled time from HIV exposure to seroconversion as an exponential hazard function, with the window period (the half-time of the exponential function) following a log-normal distribution. All model parameters were drawn randomly for each iteration of the model. All 
data distributions are listed in Table 1 . The simulation model was run 1,000 times for each of the three physician-to-patient transmission rates $(0.15 \%, 0.3 \%$, $0.6 \%$ ) at each of the seven specified physician HIV prevalence rates $(0.1 \%, 0.25 \%, 0.5 \%, 0.75 \%, 1 \%, 1.5 \%$, $2 \%$ ).

\section{PREVALENCE OF HIV INFECTION}

Data on the prevalence of HIV infection in surgeons, hospital inpatients, and various outpatient populations were obtained from a review of the literature. Most of the data come from prevalence studies of HIV infection conducted by the Centers for Disease Control and Prevention (CDC) that focus on segments of the population other than healthcare providers. ${ }^{20}$ Only one self-selected cohort of surgeons has been studied to determine HIV prevalence. ${ }^{21}$

The prevalence of HIV infection has been reported in several populations that may not be representative of the general population. The National Clinical Laboratory Survey determined overall HIV prevalence to be $0.89 \%$ ( $0.34 \%$ among women and $1.6 \%$ among men) using outpatient complete blood count laboratory specimens drawn from a sample of 5 million patients. ${ }^{20}$ The Sentinel Hospital HIV Surveillance Study, a survey of patients admitted to 26 hospitals in 21 cities in the United States and Puerto Rico, estimated an overall prevalence among all U.S. hospital admissions of $0.9 \% .22$ The American Red Cross found a $0.039 \%$ prevalence of HIV infection among first-time blood donors, a group self-selected for low HIV risk. ${ }^{23}$ In the single published study of surgeons, 2 of 3,420 orthopedists tested voluntarily at the 1991 American Academy of Orthopedic Surgeons meeting were HIV-positive, representing a prevalence of $0.06 \%$ (95\% confidence interval $\left[\mathrm{CI}_{95}\right] 0$ to $0.18 \%$ ). ${ }^{21}$ However, this cohort represented only about half of the surgeons attending the meeting. ${ }^{21}$

We ran our transmission and screening model using seven baseline surgeon prevalence rates rang. ing from $0.1 \%$ to $2 \%$. The $0.9 \%$ overall prevalence from the Sentinel Hospital HIV Surveillance Study 22 was used as the best-case estimate of inpatient HIV prevalence.

\section{INCIDENCE OF HIV INFECTYON IN SURGEONS}

The annual incidence of HIV infection among American healthcare workers is unknown. We modeled annual surgeon incidence of HIV infection based on two potential sources of transmission, occupational and nonoccupational.

The occupational transmission rate was calculated from several retrospective studies of percutaneous blood exposures of surgeons at different stages of training. ${ }^{24-26}$ Attending surgeons were estimated to experience a median of 4.2 percutaneous blood exposure episodes per 1,000 hours of surgery. ${ }^{26}$ Based on a median work-year of 720 hours of surgery, ${ }^{27}$ the average attending surgeon would have 3.1 percutaneous exposures per year. Surgical residents were estimated to incur an average of 4.2 exposures per year and medical students 1.3.25 We used these training-stage exposure rates in our analysis.

By combining several prospective studies of healthcare workers, Henderson et $\mathrm{al}^{28}$ found the risk of HIV seroconversion following an occupational percutaneous exposure to blood of an HIV-infected patient to be $0.3 \%\left(\mathrm{Cl}_{95}=0.1 \%\right.$ to $\left.0.7 \%\right)$. We modeled the annual number of instances of HIV transmission from patients to surgeons as the product of the baseline inpatient HIV prevalence, the number of percutaneous exposures per surgeon per year, and the risk of seroconversion following an occupational exposure to HIV infected blood. A survey by the American Medical Association identified 100,205 surgical attendings (including general, thoracic, colorectal, and pediatric surgeons, orthopedists, and urologists) and 21,818 surgical residents ${ }^{29}$ as of January 1,1989 . The National Resident Matching Program reported 2,438 graduating medical students entering surgical residencies in 1992.30

Nonoccupational transmission occurs mostly through high-risk behavior. We modeled nonoccupational transmission based on the prevalence of $\mathrm{HIV}$ infection in the general population because there are no primary data on the incidence of HIV infection among surgeons. First, we modeled the incidence of HIV based on the following formula:

\section{incidence $=$ prevalence $\cdot$ risk modification factor incidence conversion factor}

We included a risk modification factor because of the recently observed decrease in the incidence of HIV in several populations as knowledge of HIV transmission has grown. The 0.59 value used in this report is based on the serial calculated incidence of HIV infection in army personnel. ${ }^{31}$ We calculated an incidence conversion factor to relate the prevalence of HIV infection to the current annual incidence of this disease. The model is based on a mean incidence conversion factor of 8 (range, 4 to 12); this is our best estimate of the ratio of HIV prevalence to HIV incidence, based on changes in the patterns of acquired immune deficiency syndrome cases reported over time.

\section{HIV SCREENING}

The screening protocol we modeled is the current standard of practice as recommended by the 
$C D C .{ }^{32}$ All subjects are screened by an initial enzymelinked immunosorbent assay (ELISA) test. Those with a positive ELISA test then are confirmed with a second ELISA. If both ELISAs are positive, a confirmatory Western blot test is done. Positive HIV tests require two positive ELISA tests and a positive Western blot. All other test results are considered a negative test. A test sequence that is positive on the first ELISA but negative on the second ELISA is considered negative. Likewise, two positive ELISA assays and a negative Western blot also are considered negative. ${ }^{33}$

The sensitivity and specificity of screening protocols recently have been reviewed. ${ }^{3436}$ In our model, the first ELISA was assigned to have a sensitivity of $98.5 \%$ (range. $97 \%$ to $100 \%$ ), and a specificity of $99.5 \%$ (range, $99.4 \%$ to $99.6 \%$ ). The conditional sensitivity of the second ELISA is unchanged, while the conditional specificity of the second ELISA is $60 \%$ (range, $50 \%$ to $70 \%) .{ }^{35}$ The sensitivity and specificity of the Western blot test was taken as $96 \%$ (range, $94.5 \%$ to $97.5 \%$ ) and 99.4\% (range, $99.3 \%$ to $99.5 \%$ ) respectively. ${ }^{35}$

For incident cases, a window period was modeled. A window period is defined as the time period following HIV infection where the surgeon has not yet developed a positive antibody response to the HIV virus. Thus, during the window period, the surgeon is HIV-infected but the ELISA is negative. This window period creates a pool of false-negative subjects for each round of HIV testing. The median duration of the window period was estimated at 2.1 months $\left(\mathrm{Cl}_{95}=0\right.$ to 5.8 months).$^{37}$ As a conservative assumption, incident cases were assumed to occur at the beginning of the year at the time of screening. Thus, in the model, infected surgeons in the window period and surgeons who test falsely negative in the previous test cycle have the potential to transmit HIV infection to patients in a given year.

\section{RISK OF TRANSMISSION TO PATIENTS}

There currently are no data that provide reliable estimates of the risk of HIV transmission from an infected physician performing an invasive procedure to his or her patient. More than 15,000 patients now have been screened as part of several look-back programs assessing HIV transmission from infected physicians to patients, ${ }^{38,39}$ and no proven cases of surgeon-to-patient HIV transmission have been identified. The power to detect an infrequent event such as physician to patient transmission of HIV infection is low in this nonrandom sample of surgical patients. The risk of transmission from an HIV infected surgeon to his or her patients has been estimated as 1 per 41,600 to 1 per 416,000 cases, based on the likelihood of reciprocal exposure to blood and on physician occupational seroconversion data following occupational exposure. ${ }^{14,15,18}$

We developed a model of transmission from surgeons to patient based on the assumption of reciprocal risk of HIV transmission following a mutual percutaneous occupational exposure. Blood exposures may be mutual between surgeons and patients (ie, injuries occurring during an operation such as a scalpel wound) or nonmutual, where a physician is exposed to the patient's blood (ie, needlesticks resulting from capping accidents or from faulty use of needleboxes). Patients are at risk for acquiring HIV infection from surgeons only from mutual exposures. Among surgical residents, nonmutual exposures represent $17 \%$ of all exposures. ${ }^{24}$ The patient exposure rate was calculated by multiplying the physician injury rate by 0.83 , the mutual exposure rate for surgeons.

The patient risk of seroconversion following a mutual exposure episode is not known. It may be equal to the reported healthcare worker conversion rate; it may be lower because during a mutual exposure the physician's blood may still not be in contact with a patient (ie, even a damaged glove may protect the patient), or it may be higher since the surgeons's blood may come readily into direct contact with a patient's exposed membranes during an invasive procedure. In our primary analysis, we modeled the patient transmission rate using the healthcare worker transmission rate of $0.3 \%\left(\mathrm{CI}_{95}=0.1 \%\right.$ to $\left.0.7 \%\right),{ }^{28}$ half this rate or $0.15 \%\left(\mathrm{CI}_{95}=0 \%\right.$ to $\left.0.4 \%\right)$ and double this rate or $0.6 \%\left(\mathrm{CI}_{95}=0.3 \%\right.$ to $\left.1 \%\right)$.

\section{Risk per Procedure}

Based upon the estimated $39,129,000$ procedures performed in nonfederal, acute care hospitals in the United States in $1988,4^{40}$ we calculated the risk of physician-to-patient HIV transmission per procedure performed in the United States.

\section{Sensitivity Analysis}

This HIV transmission and screening model is based on a number of extrapolations from the literature. Sensitivity analysis is used to examine the impact of uncertainty of estimates of variables on the model results. Sensitivity analysis was incorporated into the primary analysis by using seven different physician HIV prevalence rates and by using three different physician-to-patient HIV transmission rates. In addition, all model outputs include both mean and standard deviation values. Although this method allows assessment of the interaction of model parameters on the results of the analysis, it does not provide an assessment of the dependence of the overall analysis on each data element. 
TABLE 2

Number of Patients Contracting Hiv Infection Froma Surgeon (Transmission Rate $=0.15 \%$ ) *

\begin{tabular}{lccccc}
\hline \multirow{2}{*}{$\begin{array}{l}\text { Surgeon HIV } \\
\text { Prevalence }\end{array}$} & \multicolumn{2}{c}{$\begin{array}{c}\text { Untested } \\
\text { Surgeons }\end{array}$} & & \multicolumn{2}{c}{$\begin{array}{c}\text { After First Test } \\
\text { of Surgeons }\end{array}$} \\
\cline { 2 - 3 } \cline { 5 - 6 } & Number & SD & & Number & SD \\
\hline $2 \%$ & 10.2 & 6.1 & & 0.8 & 0.5 \\
$1.5 \%$ & 7.7 & 4.2 & & 0.6 & 0.4 \\
$1 \%$ & 5 & 2.9 & & 0.4 & 0.2 \\
$0.75 \%$ & 3.7 & 2.1 & & 0.3 & 0.2 \\
$0.5 \%$ & 2.4 & 1.4 & & 0.2 & 0.1 \\
$0.25 \%$ & 1.3 & 0.7 & & 0.1 & 0.1 \\
$0.1 \%$ & 0.5 & 0.3 & & 0.1 & 0
\end{tabular}

"Data based on a surgeon-to-patient transmission rate of $0.15 \%$ per mutual exposure.

\section{RESULTS}

In the absence of testing surgeons for HIV, the model estimates that between $0.5( \pm 0.3)$ and 36.9 $( \pm 11.6)$ cases of HIV occur annually in the United States as a result of HIV transmission to patients from exposure to HIV-infected surgeons performing invasive procedures (Tables 2,3 , and 4).

After one year of a mandatory screening program with practice limitations on surgeons who test positive for HIV infection, the risk of transmission from surgeons to patients is reduced by $90 \%$ to $92 \%$ to between $0.05( \pm 0.03)$ and $3.1( \pm 1.1)$ and an additional $66 \%$ to $67 \%$ in its second year, as false-negative surgeons from the previous screening cycle are detected in the subsequent screening cycle. No further incremental benefit accrues from subsequent annual testing cycles.

The risk of physician-to-patient HIV transmission per procedure performed in the United States ranges from 0.01 to 0.9 per million procedures without testing to 0.001 to 0.08 per million procedures with testing (Table 5).

\section{Sensitivity Analysis}

Sensitivity analysis was incorporated into the primary analysis by using seven different physician HIV prevalence rates and by using three different physician-to-patient HIV transmission rates. Again, all model outputs include both mean and standard deviation values (Tables 2,3 , and 4). At a transmission rate of $0.15 \%$, we report a range of $0.5 \pm 0.3$ to $10.2 \pm 6.1$ cases of surgeon-to-patient HIV transmission, a twentyfold difference in the number of cases. At a transmission rate of $0.3 \%$, we report a range of $1 \pm 0.4$ to $19.3 \pm 8.1$ cases of surgeon-to-patient HIV transmis-
TABLE 3

Number of Patients Contracting HIV Infection from a Surgeon (Transmission Rate $=0.3 \%$ ) *

\begin{tabular}{lccccc}
\hline \multirow{2}{*}{$\begin{array}{l}\text { Surgeon HIV } \\
\text { Prevalence }\end{array}$} & \multicolumn{2}{c}{$\begin{array}{c}\text { Untested } \\
\text { Surgeons }\end{array}$} & & \multicolumn{2}{c}{$\begin{array}{c}\text { After First Test } \\
\text { of Surgeons }\end{array}$} \\
\cline { 2 - 3 } \cline { 5 - 6 } \cline { 5 - 6 } & Number & SD & & Number & SD \\
\hline $2 \%$ & 19.3 & 8.1 & & 1.6 & 0.7 \\
$1.5 \%$ & 14.5 & 6.1 & & 1.2 & 0.5 \\
$1 \%$ & 9.6 & 4 & & 0.8 & 0.4 \\
$0.75 \%$ & 7.2 & 3 & & 0.6 & 0.3 \\
$0.5 \%$ & 4.8 & 2 & & 0.4 & 0.2 \\
$0.25 \%$ & 2.4 & 1 & & 0.2 & 0.1 \\
$0.1 \%$ & 1 & 0.4 & & 0.1 & 0
\end{tabular}

*Data based on a surgeon-to-patient transmission rate of $0.3 \%$ per mutual exposure.

sion, a nineteenfold difference in the number of cases. Finally, at a transmission rate of $0.6 \%$, we report a range of $1.8 \pm 0.6$ to $36.8 \pm 11.6$ cases of surgeon-topatient HIV transmission, a twentyfold difference in the number of cases.

\section{DISCUSSION}

The desire to protect the general public from acquiring HIV infection while receiving medical care has led some to propose a policy mandating screening of certain healthcare workers for HIV infection. We have developed a model to estimate the magnitude of this risk based on HIV prevalence rates among surgeons performing invasive procedures. Although any healthcare worker is a potential source of HIV infection to a patient, we chose to focus upon surgeons because they suffer frequent percutaneous injuries while in intimate contact with patient mucous membranes, making them likely occupational recipients and transmitters of a bloodborne pathogen such as HIV. Further, the benefits of HIV screening and practice limitation for HIV-infected physicians would be expected to be greatest for this group of providers.

Several major conclusions may be drawn from our results. First, even without physician screening for HIV, the risk of a patient's becoming infected with HIV from a surgeon is very low, less than other commonly accepted risks currently faced in the healthcare setting. For instance, the risk of HIV transmission from a unit of screened blood is approximately 6.5 per 1 million units. ${ }^{41}$ Because 18 million blood units of components are transfused annually in the United States (Sandler J, American Red Cross, January 10, 1992, personal communication), 117 cases of HIV infection in blood product recipients are expected annually, assuming a transmission rate of $100 \%$ per infected blood product infused. The nation- 
TABLE 4

Number of Pattents Contracting HIV InFection proma Surgeon (Transmission Ratr $=0.6 \%$ )*

\begin{tabular}{lccccc}
\hline \multirow{2}{*}{$\begin{array}{l}\text { Surgeon HIV } \\
\text { Prevalence }\end{array}$} & \multicolumn{2}{c}{$\begin{array}{c}\text { Untested } \\
\text { Surgeons }\end{array}$} & & \multicolumn{2}{c}{$\begin{array}{c}\text { After Frst Test } \\
\text { of Surgeons }\end{array}$} \\
\cline { 2 - 3 } \cline { 5 - 6 } & Number & SD & & Number & SD \\
\hline $2 \%$ & 36.8 & 11.6 & & 3.1 & 1.1 \\
$1.5 \%$ & 27.2 & 8.6 & & 2.3 & 0.8 \\
$1 \%$ & 18.0 & 5.5 & & 1.5 & 0.5 \\
$0.75 \%$ & 13.6 & 4.2 & & 1.1 & 0.4 \\
$0.5 \%$ & 9 & 2.9 & & 0.8 & 0.3 \\
$0.25 \%$ & 4.5 & 1.5 & & 0.4 & 0.1 \\
$0.1 \%$ & 1.8 & 0.6 & & 0.2 & 0.1
\end{tabular}

"Data based on a surgeon-to-patient transmission rate of $0.6 \%$ per mutual exposure.

wide estimate of the number of cases of HIV transmissions from blood products is more than twice the highest and 10 times the lowest baseline transmissions calculated in our model without surgeon screening.

Second, a screening program cannot remove all risk from exposure of an HIV-infected physician during an invasive procedure. While the HIV screening test is one of the most sensitive and most specific diagnostic tests ever developed, even in the best hands it is not perfect. Because of lab error, test imperfections, and the window period observed in the early stages of new infections, some HIV-infected surgeons will have false-negative test results. Other physicians will become infected in the interval between test cycles. Thus, a physician screening program cannot eliminate all risk of transmission in the healthcare setting. The decision to screen surgeons, therefore, should consider the incremental impact of the program on the public's health.

Third, the model assumes that without compulsory testing, no surgeon undergoes HIV testing. Many surgeons do get tested for a variety of reasons. Thus, the model's estimates of risk in an untested state constitutes upper bounds. The incremental risk reductions brought about through a compulsory testing program also are upper bounds. Actual impact from implementation of a surgeon HIV testing and practice limitation program would result in somewhat lower benefits than those presented here.

\section{Impact of a Screening Program}

A program of screening surgeons for HIV has the potential for significant ramifications on the cost of care.

Disability insurance for surgeons, housestaff, and medical students may not provide coverage for HIV
TABLE 5

Patient Risk of Aceutrang HIV Infection per

Surgical Procedure in thrs UnIted States

\begin{tabular}{lcc}
\hline & \multicolumn{2}{c}{ Risk per Procedure } \\
\cline { 2 - 3 } Category & Untested & After First Test \\
\hline $\begin{array}{l}\text { 0.1\% prevalence } \\
0.015 \% \text { transmission rate }\end{array}$ & $0.01 / 1,000,000$ & $0.001 / 1,000,000$ \\
$1 \%$ prevalence & & \\
$0.03 \%$ transmission rate & $0.25 / 1,000,000$ & $0.02 / 1,000,000$ \\
$2 \%$ prevalence & & \\
$0.06 \%$ transmission rate & $0.9 / 1,000,000$ & $0.08 / 1,000,000$ \\
\hline
\end{tabular}

infection under current policies. Further, housestaff disability policies do not provide adequate protection for young families because the income of physicians is artificially low during residency, and disability insurance is based on actual earnings at the time of disability. Medical students generally are not covered by any disability insurance coverage despite the fact that they are involved routinely in patient care activities as part of their educational experience, where they face the risk of HIV exposure. Creating an adequate physician disability insurance fund for physicians and physicians in training is an unrecognized, and currently underfunded, cost of an HIV screening program.

Even on a nominal level, an HIV screening program for surgeons itself is somewhat costly. The $\mathrm{CDC}$ estimated the cost of testing and counseling at $\$ 47$ for an HIV-negative person and $\$ 115$ for an HIV-positive person..$^{2}$ Healthcare professionals, including surgeons, require such counseling because their questions and concerns may be more complex than those expressed by patients undergoing HIV screening. Extrapolated to the 122,000 surgical attendings and residents' in the United States, HIV testing of surgeons will cost approximately $\$ 5.7$ million per test cycle. Moreover, a screening program will cost an additional $\$ 6$ to $\$ 12$ million for lost productivity, based on a billing rate of $\$ 100 /$ hour based on one half to one full hour per screening visit.

\section{Policy Implications}

In the current environment where physicians have limited or inadequate protection from the economic impact of occupationally acquired HIV disease, physicians already may have routine HIV testing performed after occupational injuries to help prove that HIV transmission was occupationally related. These tests may help physicians seek early treatment if they are HIV-positive or consider an earlier practice limitation program. 
Because there is an economic loss engendered by a surgeon who stops operating voluntarily, there needs to be a confidential mechanism established to protect HIV-infected surgeons from any financial loss if they acquire the disease, in order to remove their financial incentive to continue to operate on patients; without such a program, the economic rewards of continued practice would provide a strong incentive for surgeons to continue to practice even though they may appreciate that they are at high risk and ought to undergo HIV testing and/or stop performing invasive procedures.

\section{Limitations}

There are several limitations to our analysis. Data for many important clinical variables are limited and of uncertain reliability and validity. Thus, extrapolations and subjective estimates were required to develop model parameters, such as the per exposure risk of transmission from physician to patient and the incidence conversion factor. Further, we did not estimate the number of additional cases that may occur from nonsurgeon sources of transmission of HIV infection in the healthcare setting, including nurses, phlebotomists, and emergency services personnel. Much of the data used in developing our model came from studies reporting data for a small number of patients relative to the actual population of interest. Further, HIV-infected patients may have been late-stage patients with high HIV viral titers as compared with the general population. This analysis includes only general surgeons and not other physicians who may perform invasive procedures (including obstetrician/gynecologists, cardiologists, and interventional radiologists). It is likely that the probability of transmission of HIV from surgeons to patients, like those of sexual transmission, may vary with stage of disease, type of exposure, and with as-yet-unidentified viral factors. In this study, however, we attempted to estimate only the average risk of transmission from surgeons to patients.

Despite extensive investigation, experts from the CDC have been unable to explain why five patients apparently were infected by one dentist in Florida, a point-source spread of the infection. This type of point-source outbreak also has been reported from breakdown of sterile technique in the transmission of bloodborne infection in other healthcare settings. ${ }^{43}$ Our model is unable to examine these cases of point-source transmission of HIV infection from surgeon to patients because this type of transmission has not been reported with any predictable frequency.

Given the uncertainty in these data, we have tried to err on the side of overestimating the potential risk to patients of acquiring HIV infection from physician exposure, or of overestimating the potential benefit from screening. For example, we included closed needle (suture) injuries during surgery as exposures despite the fact that there have been no reported cases of HIV transmission through this type of contact. We assumed that the risk for all attending surgeons is uniform, although it is possible that as surgeons advance in their career, the number of potential exposures may decrease, particularly those due to hollow-bore needles used for blood drawing or as intravenous catheters.

We assumed all incident cases occurred at the beginning of the year (limiting the exposure of patients to seropositive surgeons who converted between screening tests). We also constructed the model assuming that there was no effective prophylaxis for exposed physicians or patients. Furthermore, we developed an analysis incorporating wide ranges for all of our model input estimates. However, there still may be a high degree of error in our data. Clearly, this model will need to be reexamined as better data on key parameters become available.

We did not assess the impact of improved testing technologies on these results, nor did we examine the option to test only subgroups of surgeons for HIV infection. Finally, we did not compare the costs of this program to the costs of other public health measures designed to prevent the spread of HIV infection, such as drug treatment or needle exchange programs.

\section{SUMMARY}

The risk of an individual's acquiring HIV infection from a physician is extremely low. On a national basis, the number of patients acquiring HIV infection from a surgeon without a screening program is less than half the estimated number of patients acquiring HIV from blood products after the blood products have been screened. As a public health program, a surgeon screening program cannot reduce the magnitude of the risk to patients of acquiring HIV from a surgeon to zero. The cost of such a program would be high, and the impact of an HIV screening program would be felt throughout the healthcare system. It might lead to decreased access to care for underserved populations in areas where the HIV epidemic is most prevalent. A voluntary program excluding HIV-infected surgeons from high-risk practices may provide an additional margin of safety to patients from this low risk of HIV transmission. But adequate economic safeguards for surgeons in the form of noncancelable, reasona- 
bly priced disability insurance and/or life insurance will be important incentives for HIV-infected surgeons to limit their professional practice voluntarily.

\section{REFERENCES}

1. Centers for Disease Control. Update: transmission of HIrV infection during invasive dental procedures. MMWR 1991;40:377381 .

2. Marshall PA, O'Keefe JP, Fisher SG, Caruso AJ, Surdowski J. Patients' fear of contracting the acquired immunodeficiency syndrome from physicians. Arch Interm Med 1990;150:1501-1506.

3. Scott J. Fear of AlDS from doctors highlights views on risk. The Philadelphia Inquirer August 27, 1991;A45.

4. Eickhoff T, American College of Physicians. Statement to the Centers for Disease Control. February 21-22, 1991.

5. Scott H. The HIV-infected health care worker: another policy conundrum. Arn Intern Med 1991;166:341-343.

6. American Medical Association. AMA statement on HIV-infected physicians. January $17,1991$.

7. Sen. McKay. Florida SB 20. HIV testing. 1993.

8. Rep. Jonkiert. Delaware HB 191. An act to amend Part II, Title 16 of the Delaware Code by adding a new Chapter 29 to require that healthcare professionals be tested for AIDS and human immunodeficiency virus and that positive results be reported, that infected healthcare professionals notify their patients and be prohibited from certain practices and providing penalties for violations. 136th General Assembly, 1991.

9. Maryland SB 18. Health care providers performing exposureprone procedures: HIV testing. General Assembly Regular Session, 1992.

10. Rep. Wheeler, Rep. McDowell. New Hampshire HB 1404. An act relative to mandatory testing for healthcare providers and certain patients for communicable diseases. 1992.

11. Texas HB 1362. Relating to annual testing of health care providers for AIDS and HIV infection and to the availability of those test results; providing criminal penalty. 72nd Leg., RS. 1991.

12: Rep. Dannemeyer. U.S. Congress HR 2788. Kimberly Bergalis Patient and Health Provider Protection Act of 1991. 1991.

13. Amendment 734. Appropriations for Treasury Department and U.S. Postal Service (HR 2622). Congressional Record July 11 , 1991; $\$ 9778$.

14. Nease RF, Owens DK. Estimating the risk posed to patients of HIV-infected surgeons and dentists performing invasive procedures. Med Decis Making 1991;11:325.

15. Miike L, Ostrowsky J. HIV in the health care workplace: background paper. Washington, DC: Office of Technology Assessment; 1991.

16. Gerberding JL. Expected costs of implementing a mandatory human immunodeficiency virus and hepatitis $B$ virus testing and restriction program for healthcare workers performing invasive procedures. Infect Control Hosp Epidemiol 1991;12:443-447.

17. Russo G, La Croix SJ. A second look at the cost of mandatory human immunodeficiency virus and hepatitis $B$ virus testing for healthcare workers performing invasive procedures. Infect Control Hosp Epidemiol 1992;13:107-110.

18. Bell DM, Martone WJ, Culver DH, et al. Risk of endemic HIV and hepatitis B virus transmission to patients during invasive procedures. In: Abstracts of the Seventh International Conference on AIDS. Rome, Italy: Institution Superiore di Sanita, 1991:1. Abstract 37.

19. Box GEP, Hunter WG, Hunter JS. Statistics for Experimenters. New York, NY: John Wiley \& Sons; 1978.

20. National HIV seroprevalence surveys: summary of results. Atlanta, GA: Centers for Disease Control, 1989. U.S. Department of Health and Human Services.

21. Centers for Disease Control. Preliminary analysis: HIV seropre- valence of orthopedic surgeons, 1991. MMWR 1991:40:309-312.

22. Janssen RS, St Louis ME, Satten GA, et al. HIV infection among patients in U.S. acute care hospitals: strategies for the counseling and testing of hospital patients. $N$ Engl J Med 1992;327:445452.

23. Peterson LR, Dodd R, Dondero TJ. Methodologic approaches to surveillance of HIV infection among blood donors. Pub Health Rep 1990;105:153-157.

24. Heald A, Ransohoff D. Needlestick injuries among resident physicians. J Gen Intern Med 1990;5:389-393.

25. McGeer A, Simor A, Low D. Epidemiology of needlestick injuries in house officers. J Infect Dis 1990;162:961-964.

26. Lowensfels A, Wormser G, Jain R. Frequency of puncture injuries in surgeons and estimated risk of HIV infection. Arch Surg 1989;124:1284-1286.

27. American Medical Association. Physician Marketplace Statistics. Fall 1990. Chicago, IL: American Medical Association; 1990.

28. Henderson D, Fahey BJ, Willy $M$, et al. Risk for occupational transmission of human immunodeficiency virus type 1 associated with clinical exposures: a prospective clinical evaluation. Ann Intern Med 1990;113:740-746.

29. American Medical Association Department of Physician Data Services, Division of Survey and Data Resources. Physician Characteristics and Distribution in the U.S. Chicago, IL: American Medical Association; 1990.

30. National Resident Matching Program. NRMP Data: April 1992. Evanston, IL: National Resident Matching Program; 1992.

31. McNeill JG, Brundage JF, Gardner LI, et al. Trends of HIV seroconversion among young adults in the U.S. Army, 19851989. JAMA 1991;265:1709-1714.

32. Centers for Disease Control. Public Health Service guidelines for counselling and antibody testing to prevent HIV infection and AIDS. $M M W R$ 1987;36:509-515.

33. Centers for Disease Control. Interpretation and use of the Western Blot assay for serodiagnosis of HIV-1 infected individuals. $M M W R$ 1989; (suppl 7):1-7.

34. Schwartz JS, Dans P, Kinosian B. Human immunodeficiency virus test evaluation, performance, and use.JAMA 1988;259:2574 2579 .

35. Schwartz JS, Kinosian B, Pierskalla W, Lee H. Strategies for screening blood for human immunodeficiency virus antibody. JAMA 1990;264:1704-1710.

36. Sloand EM, Pitt E, Chiarello RJ, Nemo GJ. HIV testing: state of the art. JAMA 1991; 266:2861-2866.

37. Horsburg CR, Ou C-Y, Jason J, et al. Duration of human immunodeficiency virus infection before detection of antibody. Lancet 1989;9:637-640.

38. Centers for Disease Control. Update: investigations of patients who have been treated by HIV-infected health care workers. MMWR 1992;41:344-346.

39. Danila RN, MäcDonald KL, Rhame FS, et al. A look-back investigation of patients of an HIV-infected physician: public health implications. $N$ Engl J Med 1991;325:1406-1411.

40. National Center for Health Statistics. National Hospital Discharge Survey, 1988 . Hyattsville, MD: Department of Health and Human Services; 1990.

41. Cumming PD, Wallace EL, Schorr JB, Dodd RY. Exposure of patients to human immunodeficiency virus through the transfusion of blood components that test antibody-negative. $N$ Engl J Med 1989:321;941-946.

42. Hedlund K, Spencer J, Schalla W, Johnson L, Fehd R. Estimating public costs of HIV counseling and testing, USA Presented at the Sixth International Conference on AIDS. San Francisco, California, June 20-24, 1990

43. Polish LB, Shapiro CN, Bauer F, et al. Nosocomial transmission of hepatitis B virus associated with the use of a spring-loaded finger-stick device. N Engl J Med 1992;326:721-725. 